\title{
Analysis on Contact Stress Model of Double Circular-Arc Helical Gears based on 3D Fractal Theory
}

\author{
Yang Shufeng ${ }^{1, *}$, Liu Shijun ${ }^{1}$, Yu Wentao ${ }^{1}$, Wang Yingjie² and Qin Jianying ${ }^{3}$ \\ ${ }^{1}$ Zhengzhou Research Institute of Mechanical Engineering, China Academy of Machinery Science and Technology, Zhengzhou 450052, \\ China \\ ${ }^{2}$ School of Mechanical \&precision Instrument Engineering, Xi'an University of Technology, Xi'an 710048, China \\ ${ }^{3}$ Electrical Engineering and Information Technology, Dortmund university of technology, Dortmund D-44227, Germany
}

Received 12 August 2020; Accepted 29 November 2020

\begin{abstract}
The accurate calculation of gear contact stress is a significant parameter to judge the contact strength. It is the precondition for the investigation of gear's dynamic characteristics, fault diagnosis, and parameter optimization design. The calculation formula for the contact stress of double circular-arc helical gears is generalized from the finite element method and electrical testing, which neglects the influence of tooth surface morphology on the contact stress. In order to improve the calculation accuracy of the contact stress of the double- circular-arc helical gears, In this study, the 3D fractal contact stress of asperities on the joint surfaces was analyzed using 3D fractal theory to improve the calculation accuracy of the contact stress of the double circular-arc helical gears. Moreover, the influences of asperity deformation characteristics and sliding friction were considered. The fractal model of the normal contact stress for the double circulararc helical gears was established. Then, the model accuracy was verified by comparing the testing results, and the influences of the main parameters (fractal dimension, surface roughness amplitude, and friction coefficient) on the fractal contact model were acquired. Results show that a minimum value exists between the fractal dimension and normal contact stress. When the fractal dimension is smaller than the minimum value, the normal contact load increases with the fractal dimension. The normal contact stress increases with the surface roughness amplitude, whereas the normal contact stress reduces with the increase of the surface friction coefficient. The proposed model provides a theoretical basis for further research on the contact state of the double circular-arc helical gears and the vibration characteristics of the tooth surface.
\end{abstract}

Keywords: Double circular-arc helical gears, 3D fractal theory, Hertz contact theory, Normal contact stress

\section{Introduction}

The contact characteristics of joint surfaces have a direct bearing on the working characteristics of a mechanical system. From the microscopic perspective, a machined surface comprises numerous asperities of convex peaks and concave valleys with different geometric sizes. When two rough surfaces contact each other, the asperities will undertake the load and undergo deformation. Then, the actual contact area reduces, whereas the actual contact pressure elevates. This condition accelerates the asperity deformation and causes advanced damage. It also further generates significant influences on power transmission and sealing performance[1]. Local (Chinese) and international scholars investigated the normal contact problem of mechanical joint surfaces and established the statistical contact model [2], fractal contact model [3], finite element model [4], and multi-scale model [5]. Among them, the statistical contact model and fractal contact model are the most commonly used.

With the development of new materials and new gear tooth profiles, the working conditions that gears adopted have broad applications. Given the complexity of application conditions, the limitations of involute gears have become

*E-mail address: yangshufeng8610@163.com

ISSN: 1791-2377 @ 2020 School of Science, IHU. All rights reserved. doi:10.25103/jestr. 136.20 increasingly prominent. For instance, the fatigue pitting phenomenon can be easily generated at the node under low speed and heavy load conditions. The double circular-arc helical gears are formed through the convex arc and concave arc meshing and have been extensively applied to industrial production. Their application involves a relatively large radius of curvature, high contact strength, a constant sliding speed at different points on the tooth surface, an easy formation of oil film, and small relative error after runningin [6]. The gear contact stress of convex and concave arcs are equal after running-in, and the error can be large if the gear contact stress is solved using the traditional Hertz formula. In the national standard GB/T13799-92, the calculation formula of gear contact stress is concluded and summarized through the comparison of finite element calculation results with the experimental measurement results. Many parameters are included in the calculation process with relatively tedious solving. However, the contact between tooth surfaces belongs to the category of an indefinite boundary, and the change process of the contact area is nonlinear. Thus, the solving result can be achieved only through multiple iterations.

Following the concept of the numerical method, the nonlinear contact problem is discretized into a linear problem, thereby providing a new idea for solving the contact stress of circular arc gears. With the development of computer technology, the finite element analysis method has been applied to considerable research on gear contact stress 
[7]. This innovation further improves the precision of contact stress of circular arc gears. Yet, the micromorphology of the tooth surface is not considered in the simulation process, the type and quantity of discrete units have no unified standard, and the convergence speed is greatly related to the computer performance. The scaleless property and self-similarity of fractal theory have well solved the irregular morphological variation in the local contact region and the consistency of local morphology with the overall morphology. Moreover, the complexity and irregularity of different profiles are expressed by fractal dimension. A typical example of the fractal theory application to contact stress solving is the rough set elasticplastic contact fractal model (M-B model) established by Msajumdar and Bhushan [8]. However, the asperity deformation in the model will undergo sudden change, which does not accord with the actual deformation. The correction coefficient is proposed for the contact surface by the Hefei University of Technology [9]. Although it is a fractal model for the contact stress of involute gears, the influence of the friction factor is not considered. Given the diversity of tooth surface morphology and contact mode, the gear contact stress model based on fractal theory remains to be deeply investigated.

Following the above discussion, accurately solving the contact stress of the double circular-arc helical gears and mastering the influences of the tooth surface morphology and friction coefficient on contact stress are problems needing urgent solutions. On the basis of Hertz contact theory and improved morphological W-M function, in the current study, a 3D fractal model considering the friction coefficient was established for the contact stress of the double circular-arc helical gears by combining the macroscopic and microscopic aspects. The model effectiveness was comparatively verified, and the influences of factors, including friction on the contact stress, were analyzed to provide a reference for the subsequent bearing capacity analysis and kinetic analysis of the double circulararc helical gears.

\section{State of the art}

To master the contact and mechanical properties of rough surfaces, local and international scholars conducted various investigations on the normal contact problem of mechanical joint surfaces. Vakis [10] acquired the total sum of deformations of bases contacting the asperities within a specific range by using the statistical method. The reason was that the asperity deformation might cause base deformation. The above study also discussed the influence of the interaction order on the contact force and proposed a roughness limit. Yet, the model only involved the base deformation under Hertz contact pressure. Following the coordinate points of the double-variable W-M function, Jana [11] generated a curved fractal surface and imported it into the ANSYS software to establish models with different grades. The above research investigated the static and dynamic behaviors of the rough fractal surface when contacting the rigid plate. Yuan et al. [12] studied the fractal model of rough surface contact during the loading and unloading processes. They provided the section functions under different roughness levels in the loading process and obtained the load-contact area relation consistency in the loading and unloading processes. Prajapati [13] conducted fractal research on the contact fatigue damage through a series of double-disk experiments and found that the autocorrelation length had a positive correlation with the surface damage. Using the Y-K model, Liou et al. [14] studied the contact problem between a $3 \mathrm{D}$ cylinder and a rough spherical surface. They also comparatively analyzed the rough and spherical surface, cylindrical surface contact model, and rough plane contact model. The results showed that different contact models had similar mechanical properties. Liu [15] derived the W-M function under the spherical coordinate system, acquired the profile of the rough spherical surface, established a fractal contact model of the rough sphere, and analyzed the influences of fractal dimension and roughness. $\mathrm{Xu}$ [16] transformed the plane contact problem into a contact problem between an equivalent rough surface and a rigid, smooth surface and introduced the asperity frequency index. The above study corrected the asperity area distribution function and established a corrected 3D normal contact rigid model. The results showed that the surface contact rigidity mainly came from the asperity elastic deformation and elastic-plastic deformation, and the contribution of plastic deformation to contact rigidity was zero. The above models were constructed to investigate the point, line, and plane contacts of rough surfaces but assumed that the rough surfaces were all isotropic and the practical contact surfaces were mostly anisotropic.

Chen Qi et al. [17] put forward the contact surface correction coefficient and investigated the fractal model for the contact bearing capacity between two cylindrical surfaces and the fractal model for the normal contact rigidity of the joint surfaces of two cylinders. They also extended the application of the M-B model, established the fractal contact model for involute straight gears, and analyzed the influencing factors. Hodaei [18] acquired the statistical parameters (mean value of concave-convex peak curvature, the standard deviation of concave-convex height distribution, and area density of concave-convex height distribution) and constructed a statistical contact model for a helical gear. Ma Dengqiu et al. [19] investigated the fractal model for the sliding friction contact of variable hyperbolic circular arc cylindrical gear. Liu [20] combined the M-B contact model with the finite element method to study the torsional rigidity of a cycloidal gear. The results showed that under the constant input torque, the torsional rigidity of the cycloidal gear could be increased by increasing the fractal dimension $\mathrm{D}$ and reducing the fractal roughness $\mathrm{G}$. This process indicated that the smoother the tooth surface, the greater the torsional rigidity. Huang Kang [21] established the fractal model for two arbitrary complex surfaces, obtained the loadarea relational expression of the double circular-arc helical gear contact by using fractal theory, and constructed the 3D fractal contact model for the double circular-arc helical gears. However, the above study failed to consider the friction factor in the actual contact process or the elastic and plastic deformations in the contact region. Batsch [22] discussed the contact factors of concave and convex circular arcs influencing the circular arc gear and analyzed the influences of concave-convex tooth profile radius, the ratio of tooth profile radius to gear radius, and wheelbase error on the contact area. Markowski et al. [23] studied the influences of tooth profile overlapping ratio and translation of convex tooth profile on the contact mode. Batsch [24] applied the concave-convex circular arc contact to the bevel gear drive and used the finite element method to simulate the gear meshing. The results showed that the contact force of bevel gear with circular arc tooth profile was reduced by $22 \%$ 
Yang Shufeng, Liu Shijun, Yu Wentao, Wang Yingjie and Qin Jianying/

Journal of Engineering Science and Technology Review 13 (6) (2020) 147 - 154

compared with the traditional bevel gear. Litvin [25] determined the contact path of double circular-arc helical gear tooth surface and transient contact ellipse area and probed into the influence of gear shaft misalignment on the contact stress. Li [7] conducted a 3D dynamic meshing simulation analysis of double circular-arc helical gears and established a load-driven contact strength analysis model by using the 3D geometric contact characteristics and elastic deformation theory. The above study pointed out that the contact strength could be enhanced by enlarging the helical angle and slightly reducing the center distance. Chen [26] studied the contact stress of the double circular-arc helical gears with tooth surface deviation, used the positive clearance distribution to calculate the contact elastic deformation and local deviation caused by manufacturing error and tooth surface wear, and calculated the contact stress and meshing region under different loads and transmission errors by using the finite element method. The above scholars implemented a certain specific fundamental research work for the contact stress of double circular-arc helical gears. But, none of them considered the influence of friction factor, not to mention the influence study of tooth surface micromorphology and contact stress.

Existing research results were mainly obtained using the rough contact model of 2D and 3D mechanical joint surfaces based on fractal theory. The contact models between plane and plane, cylinder and cylinder, and cylinder and sphere were investigated by combining Hertz contact theory. This theory has been applied to the normal contact stress solving of involute gear and variable hyperbolic circular-arc cylindrical gears. However, the application of this theory in double circular-arc gears has been scarcely investigated. In the current study, fractal theory and Hertz contact theory are used to investigate the contact stress of double circular-arc helical gear contact, considering the continuous elasticplastic deformation of micro-contact bodies. Then, a fractal model is constructed for the contact stress of the double circular-arc helical gears. Finally, the influences of fractal dimension, characteristic scale coefficient and load on the actual contact area and fractal model of contact stress are analyzed.

The remainder of this study is organized as follows: Section 3 describes the tooth surface equation of the double circular-arc helical gears, confirms the correction coefficient for asperity contact area, and verifies the coefficient effectiveness, and establishes the fractal model for the normal contact stress of the double circular-arc helical gears and certifies the model accuracy. Section 4 analyzes the influences of fractal dimension, surface roughness amplitude, and friction coefficient on the normal contact stress. Section 5 summarizes the discussion and findings and provides related conclusions.

\section{Methodology}

\subsection{Tooth surface equation}

The basic tooth profile of double circular-arc helical gears consists of two circular arc segments: convex tooth and concave tooth, the radius difference $\Delta \rho$ of which is small with favorable gear tooth running-in characteristics. The contact form in the transmission is point contact, and the contact track moves along the axial direction. The principle of tooth surface formation through gear racks is adopted for the equations of both tooth surfaces of the double-double circular-arc gears. The dextrorotation convex tooth is taken for as an example, as shown in Fig. 1. Under the rack coordinate system $S_{n}\left(O_{n} X_{n} Y_{n} Z_{n}\right)$ and gear face coordinate system, the equation of convex circular arc generatrix $\Gamma_{1}$ is $r_{n}=r_{n}(\alpha)$ ( $r_{n}$ is vector), and the equation of right tooth surface of the convex tooth can be obtained through the transformation of vector coordinates as follows:

$$
\begin{aligned}
& r_{t}=r_{n}(i, \beta)^{r o t}+r i \\
& r_{1}=r_{t}(k, \varphi)^{r o t}+\left(p \varphi-\frac{l+c}{\sin \beta}\right) \cdot k
\end{aligned}
$$

Eq. (1) indicates that the generatrix rotates from the initial position around axis $x_{n}$ for $\beta$ and moves for a distance of $r_{1}$ Eq. (2) implies that the generatrix rotates around axis $z_{1}$ for $\varphi$ and moves for a distance of $p \varphi-\frac{l+c}{\sin \beta}$ Eq. (2) is written into a component form as follows:

$$
\left\{\begin{array}{c}
x_{1}=\left(\rho_{a} \sin \alpha+e+r_{1}\right) \cos \phi+\left(\rho_{a} \cos \alpha+c\right) \cos \beta \sin \phi \\
y_{1}=\left(\rho_{a} \sin \alpha+e+r_{1}\right) \sin \phi-\left(\rho_{a} \cos \alpha+c\right) \cos \beta \cos \phi \\
z_{1}=p \phi-\frac{l+c}{\sin \beta}+\left(\rho_{a} \cos \alpha+c\right) \sin \beta
\end{array}\right.
$$

where $\rho_{a}$ is the circular arc radius of the convex tooth profile. $e$ is the offset of the center of the tooth profile. $r_{1}$ is the pitch radius of the convex tooth. $c$ is the offset of the center of the tooth profile. $p_{1}$ is a helix parameter. $l$ is the offset of the center of the tooth profile and the axis of symmetry. Other symbols are annotated in the literature [6].

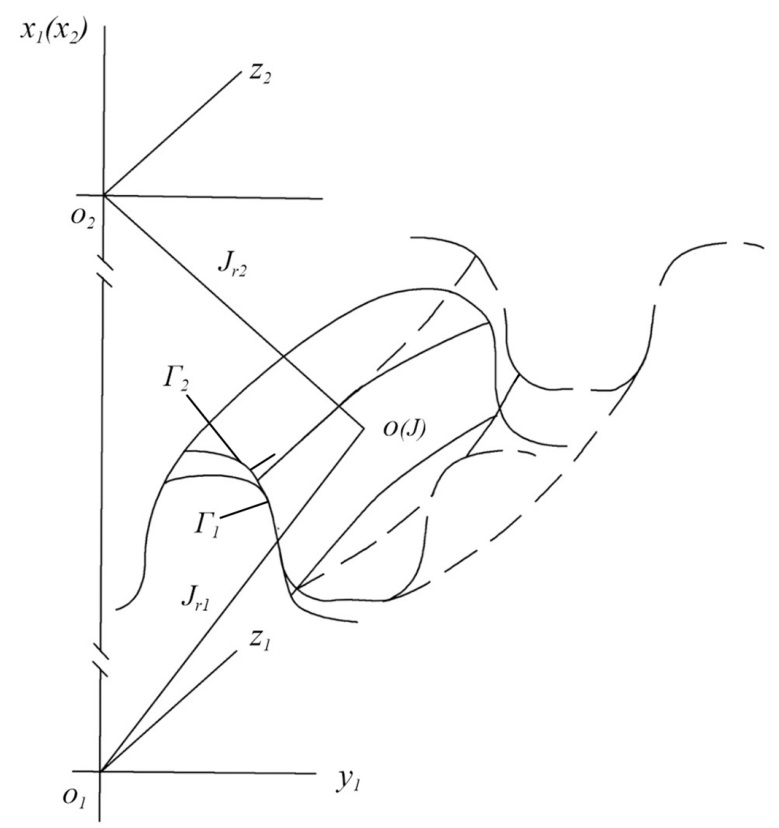

Fig. 1. Transmission diagram and coordinate system of double circulararc helical gears

Similarly, the tooth surface equation of the levorotation concave tooth meshing with the convex tooth can be solved 
Yang Shufeng, Liu Shijun, Yu Wentao, Wang Yingjie and Qin Jianying/

Journal of Engineering Science and Technology Review 13 (6) (2020) 147 - 154

by substituting the negative values of the rotation angle $\varphi$ and helix parameter.

According to the tooth surface equation of double circular-arc helical gears, the principal curvatures $k_{1}^{i}$ and $k_{2}^{i}(i=1,2)$ at the meshing point of two circular arc tooth profiles can be solved through the gear meshing principle [27] under the extremum conditions. As the direction deviation of the principal curvatures corresponding to convex and concave circular arc tooth surfaces is small, the normal curvature of the tooth surface within the coordinate plane is equal to the principal curvature at the meshing point. Then, the equivalent principal curvatures $K_{x}$ and $K_{y}$ are as follows:

$$
\left\{\begin{array}{c}
K_{x}=k_{1}^{1}+k_{2}^{1}=\frac{\rho_{f}-\rho_{a}}{\rho_{a} \rho_{f}} \\
K_{y}=k_{1}^{2}+k_{2}^{2}=\frac{\sin \alpha \sin ^{2} \beta\left[\left(r_{1}+r_{2}\right)+\sin \alpha \sin ^{2} \beta\left(\rho_{a}-\rho_{f}\right)\right]}{\left(r_{1}+\rho_{a} \sin \alpha \sin ^{2} \beta\right)\left(r_{2}-\rho_{f} \sin \alpha \sin ^{2} \beta\right)}
\end{array}\right.
$$

The comprehensive curvature radius $\rho$ of the conjugating tooth surfaces on the normal plane of double circular-arc helical gears is as follows:

$$
\rho=\frac{1}{K_{x}+K_{y}}
$$

The shape of the contact region of double circular-arc helical gears is influenced by the tooth profile parameters, materials, load, and running-in. As the two tooth surfaces present point contact, the contact region is largely approximate to elliptic shape. Following Hertz contact theory, the directions of the semi-major axis $l_{a}$ and semiminor axis $l_{b}$ of the ellipse in the contact region correspond to the directions of the tooth breadth and tooth height, respectively. In this study, they are calculated using the following formula [28]:

$$
\left\{\begin{array}{l}
l_{a}=k_{a}\left(\frac{3 P}{2 E(A+B)}\right)^{1 / 3} \\
l_{b}=k_{b}\left(\frac{3 P}{2 E(A+B)}\right)^{1 / 3}
\end{array}\right.
$$

The relationship between the two tooth surfaces is introduced as

$\cos \varphi=\frac{B-A}{B+A}$

Where $B-A=0.5\left|K_{x}-K_{y}\right|, \quad B+A=0.5\left(K_{x}+K_{y}\right)$, $\frac{1}{E}=\frac{1}{2}\left(\frac{1-\mu_{1}^{2}}{E_{1}}+\frac{1-\mu_{2}^{2}}{E_{3}}\right) \cdot K_{a}$ and $K_{b}$ are the functions of variable $\varphi$, they can be solved using the interpolation method following literature [26]. Then, the calculation formula for the ellipse area of the contact region is

$S=\pi l_{a} l_{b}$
Eqs. (4)-(7) are substituted into Eq. (8) to calculate the ellipse area in the actual contact region.

The overlap ratio $\varepsilon$ of double circular-arc helical gears. Two contact path lines exist on double circular-arc helical gear tooth surface. The axial distance from the contact point on the convex tooth surface to the contact point on the concave tooth surface is $q_{T A}$, the condition for the continuous transmission of double circular-arc helical gears is $b>q_{T A}$, and the calculation formula for the overlap ratio of the gears is expressed as follows:

$\varepsilon=\frac{b+q_{T A}}{p_{x}}$

where $b$ is the tooth breadth; $p_{x}$ is the axial distance of the contact points on the adjacent concave and convex tooth surfaces, and the concrete formula refers to the literature [6].

Length $L$ of the contact line. Length $L$ of the contact line on double circular-arc helical gears is related to the gear breadth $b$ and overlap ratio $\varepsilon$.

$L=\frac{b \varepsilon}{\cos \beta}$

\subsection{Determination of correction coefficient for asperity contact area}

The contact process of double circular-arc helical gears can be macroscopically identified as the internal contact of two arc surfaces. However, from the microscopic angle, it is considered the external contact of the two joint surfaces. When the two contacts are cylinders, the number of contacting asperities within the contact surface is reduced. Consequently, the $\lambda_{c}$ coefficient should be introduced to correct the asperity area distribution function [29]. It can be obtained by introducing 3D fractal contact theory when two planes contact each other. The relation expression between the area distribution function $n(a)$ of asperities on the contact surface and the maximum contact area $a_{1}$ is

Assume that double circular-arc helical gears are of uniform texture and isotropy, then the distribution function satisfies the following equation:

$n(a)=\frac{1}{2} \lambda_{c}(D-1) a_{1}^{\frac{D-1}{2}} a^{\frac{D+1}{2}} \quad 0 \leq a \leq a_{1}, 2<D<3$

where $D$ is the fractal dimension, and $a_{1}$ is the maximum contact area of asperities.

Following the surface correction coefficient $\lambda_{c}$ given in the literature [11] and considering the influences of the curvature radius of the contact point of tooth surfaces and the circular arc radius of the tooth profile, the relational expression of $\lambda_{c}$ is constructed as follows:

$\lambda_{c}=\left(\frac{S}{\sum S}\right)^{1 / \rho}$

When double circular-arc helical gears are installed in accordance with the reference center distance,

$\sum S=2 \pi\left(r_{1}+r_{2}\right) L$ 
where $r_{1}$ and $r_{2}$ represent the pitch radiuses of double circular-arc meshing gears, and $L$ is the length of line of action. Eqs. (8)-(11) and (13) are substituted into Eq. (12) to obtain

$$
\lambda_{c}=\left(\frac{l_{a} l_{b} \cos \beta}{2\left(r_{1}+r_{2}\right) b \varepsilon}\right)^{\left(K_{x}+K_{y}\right)}
$$

\subsection{Reasonability analysis of coefficient $\lambda_{c}$}

By analyzing the surface contact coefficient $\lambda_{c}, \lambda_{c}$ is the function related to curvature radius and the load of double circular-arc helical gears, and the curvature radius is the function related to normal module and load. $E=2 \times 10^{11} \mathrm{pa}, b=80 \mathrm{~mm}, P=1000 \mathrm{~N}, z_{1}=21, u=1$, and $\beta=11.478^{\circ}$ are taken, and the $\lambda_{c}-m_{n}$ curve chart is obtained through the numerical calculation, as shown in Fig. 2. $\lambda_{c}$ increases with the normal modulus $m_{n}$ of the meshing gears. As the radius of the circular-arc tooth profile is directly proportional to the normal modulus $m_{n}$ and with the increase of $m_{n}$, the contact type can be approximately regarded as two-plane contact, and the $\lambda_{c}$ value is approximately equal to 1 . When the normal modulus is fixed, the $\lambda_{c}-P$ curve chart can be obtained, as shown in Fig. 3 . Under the condition of $P=0$, the two gears do not contact, and $\lambda_{c}$ is $0 . \lambda_{c}$ increases with the $P$ value, but the change amplitude is small. Accordingly, the calculation formula for the contact area correction coefficient is reasonable, laying a foundation for solving the normal contact stress of double circular-arc helical gears in the later phase.

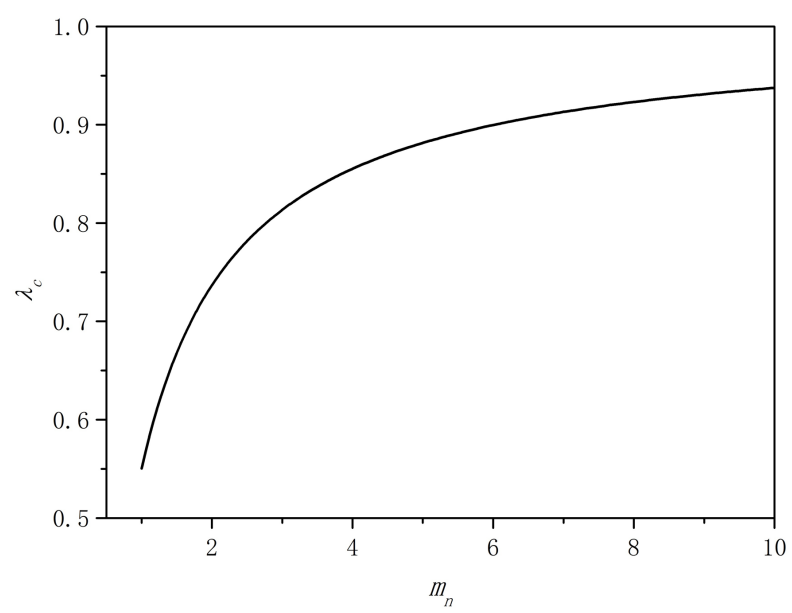

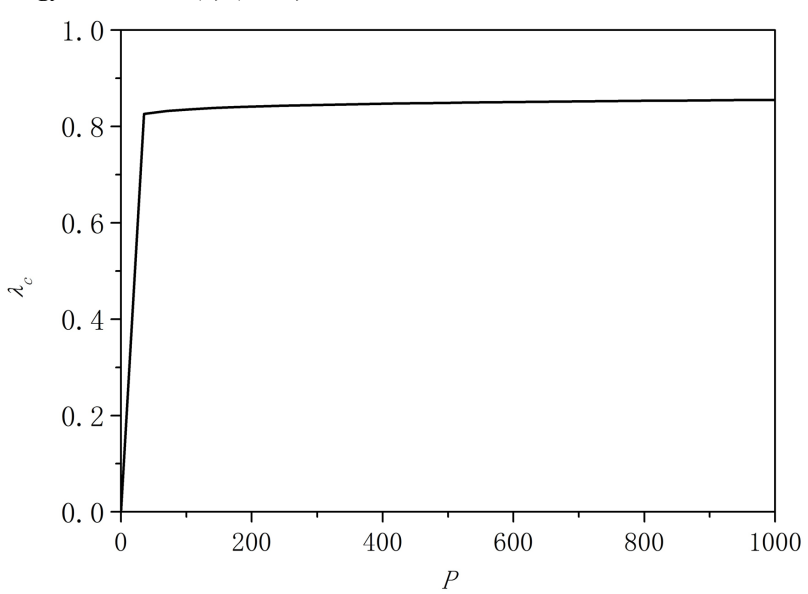

Fig. 3. Relational graph of $P$ and $\lambda_{c}$

\subsection{D fractal asperity contact model}

The continuity research on the asperity deformation on the 3D rough surface of two contacts is followed in this study. The influences of asperity deformation characteristics and sliding friction are considered under the load action. The contact deformation region of $3 \mathrm{D}$ asperities is divided into four deformation process - plastic deformation, deformation of the first elastic-plastic region, deformation of the second elastic-plastic region, and elastic deformation - on the basis of the critical areas $a_{p t 1}$ and $a_{p t 2}$ of the first and second elastic-plastic deformation regions and the elastic critical area $a_{c}$. The relational expressions of asperity contact area $A$ with plastic deformation load $P_{p}$, deformation load $P_{e p 1}$ of the first elastic-plastic region, deformation load $P_{e p 2}$ of the second elastic-plastic region, and elastic deformation load $P_{e}$ are respectively established.

$$
\begin{gathered}
P=\int_{0}^{a_{e p 1}} P_{p} n(a) d a+\int_{a_{e p 1}}^{a_{e p 2}} P_{e p 1} n(a) d a+ \\
\int_{a_{e p 2}}^{a_{c}} P_{e p 2} n(a) d a+\int_{a_{e}}^{a_{1}} P_{e} n(a) d a
\end{gathered}
$$

When $a_{1}>a_{c}$ and $D \neq 2.5$, the dimensionless equation of the normal load and contact area is expressed as follows Eq. (16).

Similarly, when $a_{1}>a_{c}$ and $D=2.5$, the dimensionless equation of the normal load and contact area is expressed as follows :Eq. (17)

Fig. 2. Relational graph of $m_{n}$ and $\lambda_{c}$

$$
\begin{aligned}
P_{c}^{*}\left(A_{r}^{*}\right)= & \lambda \varphi h_{1} 110^{\frac{3-D}{4-2 D}} A_{r}^{* 0.5 D-0.5} a_{c}^{* 1.5-0.5 D}+\frac{1.4}{3}\left(\frac{33 k_{\mu} \phi}{40}\right)^{0.474} h_{2} 2^{3.393-0.289 D} \pi^{0.263 D-1.052}(\ln \gamma)^{0.263} \\
& G^{* 0.256 D-1.052} A_{r}^{* 0.5 D-0.5} a_{c}^{* 2.026-0.763 D}\left[6^{\frac{2.026-0.763 D}{2-D}}-110^{\frac{2.026-0.763 D}{2-D}}\right]+\frac{1.03}{3}\left(\frac{33 k_{\mu} \phi}{40}\right)^{0.15} h_{3} \\
& 2^{5.25-0.775 D} \pi^{0.425 D-1.7}(\ln \gamma)^{0.425} G^{* 0.85 D-1.7} A_{r}^{* 0.5 D-0.5} a_{c}^{* 2.35-0.925 D}\left[1-6^{\frac{2.35-0.925 D}{2-D}}\right]+\frac{1}{3} \pi^{0.5 D-2} \\
& 2^{6.5-1.5 D} h_{4}(\ln \gamma)^{0.5} G^{* D-2} A_{r}^{* 0.5 D-0.5}\left[A_{r}^{* 2.5-D}-a_{c}^{* 2.5-D}\right]
\end{aligned}
$$




$$
\begin{aligned}
P_{c}^{*}\left(A_{r}^{*}\right)= & 3^{0.25} \lambda \varphi 110^{-0.5} A_{r}^{* 0.75} a_{c}^{* 0.25}+0.7\left(\frac{33 k_{\mu} \phi}{40}\right)^{0.474} 2^{1.9205} \pi^{-0.3945}(\ln \gamma)^{0.263} G^{* 0.263} A_{r}^{* 0.75} \\
& a_{c}^{* 0.1185}\left[6^{-0.237}-110^{-0.237}\right]+0.515\left(\frac{33 k_{\mu} \phi}{40}\right)^{0.15} 2^{2.5625} \pi^{-0.6375}(\ln \gamma)^{0.425} G^{* 0.425} A_{r}^{* 0.75} \\
& a_{c}^{* 0.0375}\left[1-6^{-0.075}\right]+0.125 \pi^{-0.75} 2^{4.75}(\ln \gamma)^{0.5} G^{* 0.5} A_{r}^{* 0.75} \ln \frac{A_{r}^{*}}{a_{c}^{*}}
\end{aligned}
$$

where $P_{c}$ is the normal load when two rough planes contact each other, $D$ is the fractal dimension, $P_{c}^{*}=P_{c} /\left(E A_{a}\right)$, $\lambda=H / \sigma_{s}, H$ is the hardness of the soft material, $\phi=\sigma_{s} / E, A_{r}^{*}=A_{r} / A_{a}, A_{a}$ is the nominal contact area, which can be confirmed through the sampling length $l$. $A_{a}=l^{2}$, where $l=1 m m \cdot a_{c}^{*}=a_{c} / A_{a}$, and $a_{c}$ is the critical area when a single asperity experiences elastic-plastic deformation, $a_{c}=2^{\frac{3 D-11}{2-D}}\left(\frac{33 k_{\mu} \phi}{40}\right)^{\frac{2}{2-D}} \pi^{\frac{4-D}{2-D}}(\ln \gamma)^{\frac{1}{D-2}} G^{2}$, and $G^{*}$ is the roughness amplitude function, $G^{*}=G / \sqrt{A_{a}}$, $h_{1}=\frac{D-1}{3-D}, h_{2}=\frac{D-1}{2.026-0.763 D}, h_{3}=\frac{D-1}{2.35-0.925 D}$, and $h_{4}=\frac{D-1}{2.5-D}$.

The meshing only at the node is considered in the calculation of the contact load of double circular-arc tooth surfaces. The contact problem of the gear joint surfaces is analyzed by combining the gear surface contact coefficient proposed in the literature [6], where $P^{*}$ is the dimensionless total normal load of gear joint surfaces:

$$
P_{c}^{*}=\frac{\left(\frac{P^{*} A_{a} E}{\lambda_{c}}\right)^{\frac{1}{1+2 \rho}}}{A_{a} E}
$$

\subsection{Comparison of model calculation results}

In this study, the correctness of the theoretical model is further verified. The contact force of double circular-arc gears is calculated using the fractal model, and the experimentally measured value and the value solved through the traditional formula are compared.

Following the theoretical analysis and measurement of the surface microtopography and roughness of the machined parts and components in the literature [13], the gear surface roughness is taken as 0.8 , its fractal dimension is $D=2.45$, and $G=1.2 \times 10^{-9}$. Moreover, the contact stress can be calculated through the ratio of the total contact load to the nominal area.

A pair of standardly installed double circular-arc helical gears is used in the calculation. The material texture is medium carbon quenched 45 steel. Its yield strength, hardness, comprehensive elasticity modulus, and Poisson's ratio are $\sigma_{s}=550 \mathrm{MPa}, H=48.5 \mathrm{HRC}, E=209 \mathrm{GPa}$, and $\mu=0.3$, respectively. The other gear parameters are seen in the literature [21]. By combining the previous fractal calculation formula, the above data and calculation results are all imported into the MatLab program compiled according to the above formula. The real contact area of the two gears is solved on the basis of the given load, and their contact stress during meshing is further calculated. The test value of the contact stress of double circular-arc helical gears is adopted from the literature [30], and the experimentally measured mean values for five pairs of gears (lubricant coefficient not considered) are listed in Table 1. The value calculated by the established fractal model for the contact stress of double circular-arc helical gears deviates from the experimental value by $5.54 \%$.

Table 1. Comparison chart of contact stress values calculated by different methods and experimental value

\begin{tabular}{c|c|c|c}
\hline & $\begin{array}{c}\text { Experimental } \\
\text { measurement }\end{array}$ & $\begin{array}{c}\text { Traditional } \\
\text { formula }\end{array}$ & $\begin{array}{c}\text { 3D fractal } \\
\text { model }\end{array}$ \\
\hline $\begin{array}{c}\text { Contact } \\
\text { stress } \\
\text { (MPa) }\end{array}$ & 723 & 755 & 763.12 \\
Error (\%) & 0 & 4.43 & 5.54 \\
\hline
\end{tabular}

\section{Result analysis and discussion}

The fractal surface contact model of the isotropic double circular-arc helical gears is established by combining Eqs. (11)-(18) to explore the relationship between dimensionalreal contact area and normal contact load. The model is simulated via the MatLab program, and its influence factors are analyzed.

The parameter values are indicated as follows: fractal dimension is $D=[2.2,2.8]$; surface roughness amplitude is $G^{*}=\left[10^{-8}, 10^{-12}\right]$; sliding friction factor is $\mu=[0.2,0.9]$; material characteristic parameters are taken as $\phi=2.5$; hardness coefficients are $\lambda=2.8$, and the parameters of the mutually meshing double circular-arc helical gears are calculated using the above values.

\subsection{Influence of fractal dimension $D$ on dimensional- normal contact load $P^{*}$}

The graphic plotting of the results of dimensional-normal contact load $P^{*}$ is conducted using the logarithmic coordinates plotting method to realize a more intuitive data comparison. The relation curve of fractal dimension $D$ and dimensional-normal contact load $P^{*}$ is shown in Fig. 4. The normal contact load is directly proportional to the real contact area at the initial position, but it is reduced and then increased with the increase of the fractal dimension. Through further detailed solving, the 3D surface charts and contour maps of fractal dimension $D$, dimensional-real contact area $A_{r}$, and dimensional-normal contact load $P^{*}$ are drawn. A minimal value (approximately $D=2.47$ ) exists between the fractal dimension and normal contact load, which is consistent with the conclusion drawn in the literature [29]. As the fractal dimension $D$ increases, the contact area $A_{c}$ in the critical elastic deformation phase is reduced. Thus, the number of micro-contact points under elastic contact status increases. Specifically, $A_{r}$ is increased, 
Yang Shufeng, Liu Shijun, Yu Wentao, Wang Yingjie and Qin Jianying/

Journal of Engineering Science and Technology Review 13 (6) (2020) 147 - 154

whereas the contact load $P^{*}$ is reduced. When $D$ is further increased, the contact area $A_{r}$ is reduced. However, as the surface profile is more refined, an increasing number of contact points are observed in the plastic and elastic-plastic zones, which results in the reduction of $A_{r}$, and increase of contact load $P^{*}$
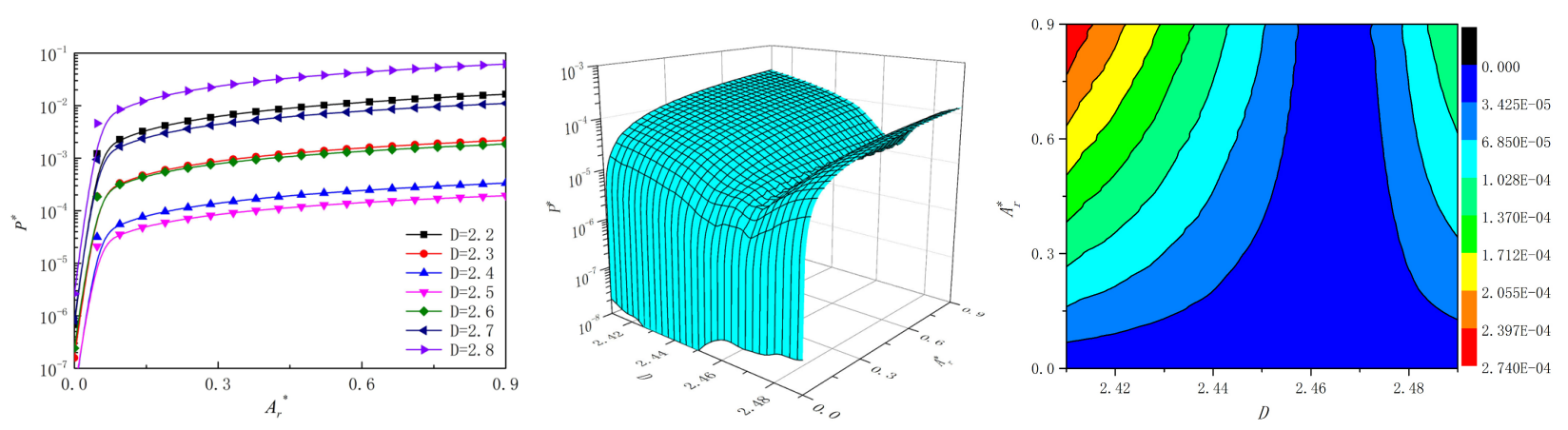

Fig. 4. Relation chart of fractal dimension $D$ and dimensional-normal contact load $P^{*}\left(G^{*}=10^{-10}, \varphi=2.5, \mu=0.3, \lambda=2.8\right)$

\subsection{Influence of surface roughness amplitude $G^{*}$ on dimensional-normal contact load $P^{*}$}

The relation chart of surface roughness amplitude $G^{*}$ and dimensional-normal contact load $P^{*}$ is shown in Fig. 5. The dimensional-normal contact load is increased with the surface roughness amplitude. Under the same fractal dimension, material characteristic coefficient, and hardness coefficient, the contact area is reduced, whereas the normal load is increased with the reduction of surface roughness amplitude.

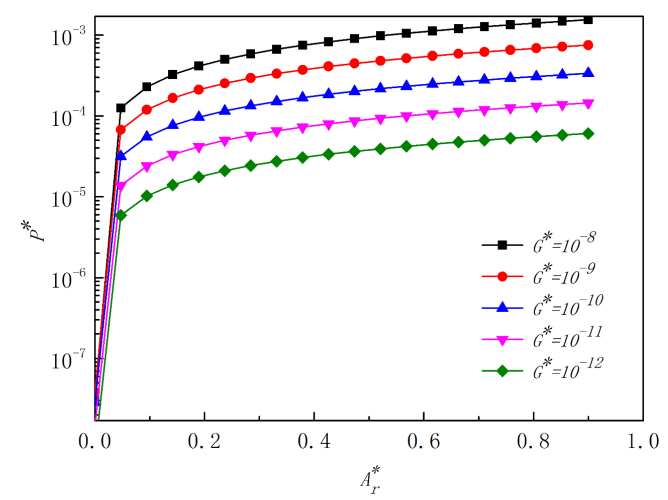

Fig. 5. Relation chart of fractal dimension $G^{*}$ and dimensional-normal contact load $P^{*}(D=2.6, \varphi=2.5, \mu=0.3, \lambda=2.8)$

\subsection{Influence of sliding friction coefficient $\mu$ on}

dimensional-normal contact load $P^{*}$

The relation curve of sliding friction coefficient $\mu$ and dimensional-normal contact load $P^{*}$ is shown in Fig. 6. The dimensional-normal contact load is reduced with the increase of the sliding friction coefficient. The reason is that the increasing sliding friction coefficient enlarges the frictional force between contacts, increases the deformation amount, enlarges the real contact area, and then reduces the normal contact load. The sliding friction coefficient has a minor influence on the dimensional-normal contact load.

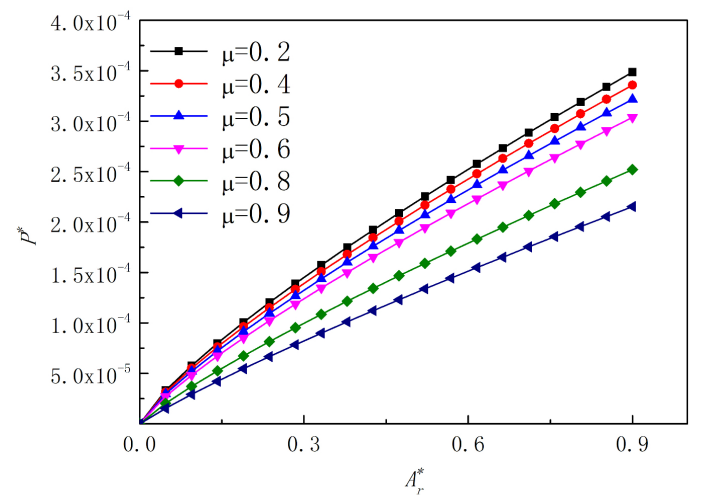

Fig. 6. Relation graph of sliding friction coefficient $\mu$ and dimensional-normal contact load $P^{*}\left(D=2.6, \varphi=2.5, G^{*}=10^{-10}, \lambda=2.8\right)$

\section{Conclusion}

For an accurate calculation of the contact stress of double circular-arc helical gears, a fractal model was established, and the influences of the fractal dimension, roughness amplitude coefficient, friction coefficient, and normal contact stress were analyzed. The following conclusions were drawn:

(1) The contact stress was related to the radiuses of the concave and convex arcs and the curvature radius of the contact point besides the fractal dimension and roughness amplitude coefficient of the joint surfaces. The constructed contact he conditions at the junction between double circular-arc helical gear tooth surfaces.

(2) The analysis results proved the existence of a fractal dimension, which resulted in the minimal value of the normal contact load. The normal contact load was directly proportional to the surface roughness amplitude coefficient but inversely proportional to the sliding friction coefficient. Moreover, the influence of the sliding friction coefficient on the contact stress was limited.

(3) The contact stress of double circular-arc gears could be improved by reducing the sliding friction coefficient and the amplitude coefficient of the tooth surface roughness.

In this study, 3D fractal theory was combined with Hertz contact theory. The whole elastic, elastic-plastic, and plastic deformation process of asperities on the joint surfaces was 
Yang Shufeng, Liu Shijun, Yu Wentao, Wang Yingjie and Qin Jianying/

Journal of Engineering Science and Technology Review 13 (6) (2020) 147 - 154

taken into account, and a fractal contact model considering the sliding friction factor was established for double circular-arc helical gears. In the establishment and analysis of the contact model, the rough joint surfaces were assumed isotropic. However, they are anisotropic under most circumstances. Therefore, the contact stress model of anisotropic contact surfaces could be discussed in further investigations to realize the practical guiding significance. New parameters could also be introduced to correct the asperity area distribution function and further improve the accuracy of the normal contact stress of double circular-arc helical gears.

\section{Acknowledgements}

The authors are grateful for the support provided by the National Science Foundation of China (Grant Nos. 51775516) and the National Key R\&D Program of China (Grant No. 2018YFB2001700).

This is an Open Access article distributed under the terms of the Creative Commons Attribution License

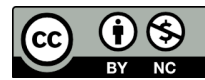

\section{References}

1. Mchale, J. P., Garimella, S. V., "Nucleate boiling from smooth and rough surfaces-Part 2: Analysis of surface roughness effects on nucleate boiling". Experimental Thermal and Fluid Science, 44, 2013, pp.439-455.

2. Xiao, H. F., Sun, Y. Y., "On the normal contact stiffness and contact resonance frequency of rough surface contact based on asperity micro-contact statistical models". European Journal of Mechanics A/Solids, 75, 2019, pp. 450-460.

3. Xu, K., Yuan, Y., Chen, J. J., "The effects of size distribution functions on contact between fractal rough surfaces". AIP Advances, 8(7), 2018, pp.075317.

4. Yan, Y. Y, Zhai, J. Y., Gao, P. X., Han, Q. K., "A multi-scale finite element contact model for seal and assembly of twin ferrule pipeline fittings". Tribology International, 125, 2018, pp.100-109.

5. Ekberg, A., Palsson, B., "Multiscale modelling of train-track interaction phenomena with focus on contact mechanics". Wear, 430, 2019, pp.393-400.

6. Lu, X. Z., Shan, j. K., "Engagement of circular arc gear". Beijing: Machinery Industry Press, China, 2003, pp.20-60.

7. Li, C. C., Chen, D. H., Li, Y. G., Mu, X. K., "Study on the meshing feature and contact strength of WN gear driving in load bearing". Applied Mechanics and Materials,229-231, 2012, pp.406-409.

8. Majumdar, A., Bhushan, B., "Fractal model of elastic-plastic contact between rough surfaces". Journal of Tribology, 113(1),1991, pp. 111.

9. Chen, Q., Zhao, H., Huang, K., “Application research on gear contact stress analysis by fractal theory”. China Mechanical Engineering, 21(9), 2010, pp.1014-1017.

10. Vakis, A. I., "Asperity Interaction and substrate deformation in statistical summation models of contact between rough surfaces". Journal of Applied Mechanics, 81(4), 2014, pp. 1-10.

11. Jana, T., Mitra, A., Sahoo, P., "Contact behavior of functionally graded fractal surface". Journal of Materials: Design and Applications, 234(10), 2020, pp. 146442072094225.

12. Yuan, Y., Xu, K., Zhao, K., “A fractal model of contact between rough surfaces for a complete loading-unloading process ". Journal of Mechanical Engneering Science, 234(4), 2020, pp.2923-2935.

13. Prajapati, D. K, Tiwari, M., "Experimental analysis of contact fatigue damage using fractal methodologies". Wear, 450-451, 2020, pp. 203262.

14. Liou, J. L, Lin, J. F., “A modified fractal micro-contact model developed for asperity heights with variable morphology parameters". Wear, 268(1), 2010, pp.133-144.

15. Liu, Y., Wang, Y. S., Chen, X., "A spherical conformal contact model considering frictional and microscopic factors based on fractal theory". Chaos Solitons \& Fractals, 111, 2018, pp.96-107.
16. Xu, K., Yuan, Y., Zhang, L. H., "A Normal Contact Stiffness Model of Joint surface Based on Fractal Theory". Computer Modeling in Engineering \& Sciences, 119(3), 2018, pp.459-480.

17. Chen, Q., Huang S. W., Zhang Z., "Research on fractal contact model for contact carrying capacity of two cylinders' surfaces considering friction factors". Journal of mechanical Engineering, 52(07), 2016, pp.114-121.

18. Hodaei, M., Rabbani, V., Maghoul, P., Bahari, A., Farhang, K., Wu, N., "Analytical modeling of contact mechanics of helical gear tooth by considering surface roughness". Journal of Adhesion Science and Technology, 34(21), 2020, pp.2387.

19. Ma, Q. D., Hou, L., Wei, Y. Q., "Sliding friction contact mechanics model of the involute arc cylindrical gear based on fractal theory". Journal of mechanical Engineering, 52(15), 2016, pp.121-127.

20. Liu, Z. F., Zhang, T., Wang, Y. D., Yang, C. B., Zhao, Y. S., "Experiment studies on torsional stiffness of cycloid gear based on machining parameters of tooth surfaces". International Journal of Precision Engineering and Manufacturing, 20 (4), 2019, pp.10171025.

21. Huang, K., Han, S. B., Yang,H. D., "Contact model of double circular arc gear based on fractal theory". Applied Mechanics and Materials, 43(4), 2011, pp.414-419.

22. Batsch, M., Markowski, T., "Influence of Novikov convexoconcave gear parameters on contact pattern". Scientific Journal of Silesian University of Technology. Series Transport, 89(4), 2015, pp.89-99.

23. Markowski, T., Batsch, M., "Tooth contact analysis of Novkov convexo-concave gears". Advances in Manufacturing Science and Technology, 39(1), 2015, pp.53-69.

24. Batsch, M., "Mathematical model and tooth contact analysis of convexo-concave helical bevel Novikov gear mesh". Mechanism and Machine Theory, 149, 2020, pp.103842.

25. Litvin, F. L., Lu, J., "Computerized simulation of generation, meshing and contact of double circular-arc helical gears". Mathematical \& Computer Modelling, 18(5), 1993, pp.31-47.

26. Chen, D. H., Zhang, Z. W., "Simulation analysis of contact pattern and strength of WN gears having tooth surface deviations". Advanced Materials Research, 479-484,2012, pp.944-948.

27. Litvin F. L., Fuentes, A., "Gear geometry and applied theory". Newyork: Cambridge University Press, America, 2004, pp.245260

28. Ge, S. R., Zhu, H., "Fractal of Tribology". Beijing: Machinery Industry Press, China,2005, pp. 150-190

29. Pan, W. J., Li, X. P., Wang, X., "Contact mechanics of elasticplastic fractal surfaces and static friction analysis of asperity scale". Engineering Computations, 2020, ahead-of-print(ahead-of-print).

30. Wu B. L., Shao J. H., "Fatigue test of double circular arc gear". Mining and Machinery, 5, 1993, pp. 21-23. 